\title{
Necessary and Sufficient Conditions for Integral Representations of Wightman Functionals at Schwinger Points
}

\author{
H. J. Borchers and J. Yngvason \\ Institut für Theoretische Physik, Universität Göttingen, D-3400 Göttingen, \\ Federal Republic of Germany
}

\begin{abstract}
Given a set of Wightman functions one would like to associate to it a field on Euclidean space admitting a simultaneous diagonalization. We investigate when this can be done in such a way that the Schwinger functions are the expectation values of this commutative field with a bounded metric operator commuting with the field. This requires as a tool the characterization of those linear functionals on the symmetric tensor algebra over a space of test functions which can be represented by complex measures on the corresponding space of distributions.
\end{abstract}

\section{Introduction}

The representation of Schwinger functions as moments of a measure on a space of distributions has, in the past few years, proved to be a useful tool in constructive quantum field theory. It is therefore interesting to ask to what extent such a representation can be derived from the Wightman axioms.

Starting from these axioms one can go by analytic continuation to the Schwinger points (points with real space and imaginary time components and no two arguments coinciding) and obtain the Wightman functions at Schwinger points. These functions are totally symmetric in their arguments but not defined at coinciding points. Therefore these Wightman functions at Schwinger points can be viewed as a linear functional on the symmetric tensor algebra of test functions, which is not everywhere defined but only on a subspace of this algebra. An extension of this functional to the whole algebra we will call a Schwinger functional.

By using the Hahn-Banach theorem one sees that such an extension is by no means unique. On the other hand this freedom does not affect the physics in the Minkowski world so that one can use it and try to enforce additional properties for convenience. We will try to find an extension such that the Schwinger functional is given by a (complex) measure. This property is automatically fulfilled by functionals on an abelian $C^{*}$-algebra, but not for general abelian algebras, due to 
pathologies associated with unbounded operators and which one should avoid if possible.

Such a programme leads us to the following two questions: Which functionals on the tensor algebra can be represented by measures? When can a functional on a subspace be extended to a functional on the whole space having the property of the first question? Since we are allowing complex measures we should explain the reasons why we do not treat the same problem with positive measures. The answer to the corresponding first question has previously been given by us [1] and independently by Challifour and Slinker [2] and also by Hegerfeldt [3]. A necessary and sufficient criterion is the so-called strong positivity of the functional. This is a condition which can be characterized neither topologically nor algebraically which makes it rather hopeless to find useful criteria for the corresponding second question. But quite apart from these technical difficulties we do not see any reason why every Wightman theory should have this positivity property. For instance it is clear that a functional representable by a positive or even real measure gives rise to a Wightman theory with invariance under time reversal (see e.g. [4]). If one believes in the existence of theories which do not have this symmetry one must therefore stick to complex measures.

In this paper we give a characterization of functionals on the symmetric tensor algebra admitting a representation by a measure. It turns out that these functionals are characterized purely by a continuity property (Theorem 3.7 ). Therefore given a Wightman functional at Schwinger points we can find an extending Schwinger functional representable by a measure if this continuity property is shared by the given functional. This continuity property will then be translated into familiar language and it means essentially: When $k$ of the Schwinger points come together in any $n$-point function then they produce a singularity which is independent of the remaining $n-k$ points, as long as they stay apart. This is a property which seems to be closely related to the existence of a Wilson-Zimmermann expansion $[5,6]$.

The contents of this paper are organized as follows: In the next section the tensor algebra is embedded in a larger algebra which carries a natural topology and the complex measures of interest are shown to correspond uniquely to the continuous linear functionals on this algebra. In Section 3 the restriction of this topology to the tensor algebra is written in terms of conventional seminorms. Section 4 contains some mathematical results which put this topology in a new perspective and in Section 5 we apply the results of Section 3 to Wightman functionals at Schwinger points.

For the sake of convenience, everything is carried through for the test function space $\mathscr{S}$, but the results hold also for other spaces such as $\mathscr{D}$ or the Jaffe spaces. Important is essentially only the nuclearity of the space.

\section{Measures on $\mathscr{S}_{\mathbf{R}}^{\prime}$ as Continuous Functionals on a Function Algebra}

Throughout this paper $\mathscr{S}$ resp. $\mathscr{S}_{n}$ stands for complex Schwartz space over $\mathbb{R}^{d}$ resp. $\mathbb{R}^{d \cdot n}$. The spaces of real test functions resp. tempered distributions over $\mathbb{R}^{d}$ will be denoted by $\mathscr{S}_{R}$ resp. $\mathscr{S}_{R}^{\prime}$.

The symmetric tensor algebra over $\mathscr{S}$ is the direct sum

$$
S(\mathscr{S})=\bigoplus_{n=0}^{\infty} S_{n}(\mathscr{S})
$$


where $S_{0}(\mathscr{S})=\mathbb{C}$ and $S_{n}(\mathscr{S})$ is the (completed) $n$-th symmetric tensor power of $\mathscr{S}$ equipped with the usual topology inherited from $\mathscr{S}_{n}$. In the direct sum topology, denoted by $\tau, S(\mathscr{S})$ is then a commutative, complete nuclear *-algebra, where the *-operation is defined as complex conjugation.

The dual space $S(\mathscr{S})^{\prime}$ consists of all sequences $T=\left(T_{0}, T_{1}, \ldots\right)$ with $T_{0} \in \mathbb{C}$ and $T_{n} \in \mathscr{S}_{n}^{\prime}$ such that $T_{n}$ is invariant under permutations of the arguments.

The algebra $S(\mathscr{S})$ may also be considered as the algebra of polynomial functions on $\mathscr{S}_{R}^{\prime}$ : Every $\omega \in \mathscr{S}_{R}^{\prime}$ defines uniquely a continuous real character $\chi_{\omega}=$ $(1, \omega, \omega \otimes \omega, \ldots) \in S(\mathscr{S})^{\prime}$ and we may identify an element $a \in S(\mathscr{S})$ with the function

$$
a(\omega) \equiv \chi_{\omega}(a) .
$$

In this way, the components $a_{n}$ of $a$ in $S_{n}(\mathscr{S})$ correspond to the homogeneous functions of order $n$ :

$$
a_{n}(\lambda \omega)=\lambda^{n} a_{n}(\omega) \text { for all } \lambda \in \mathbb{R} .
$$

The object of our investigation is the question when a functional $T \in S(\mathscr{S})^{\prime}$ has a representation of the form

$$
T(a)=\int a(\omega) d \mu_{\omega}
$$

where $d \mu_{\omega}$ is a complex measure on $\mathscr{S}_{R}^{\prime}$. For the definition of a measure one must specify a $\sigma$-algebra, but this does not pose any problems in the case of $\mathscr{S}_{R}^{\prime}$ : All the natural $\sigma$-algebras at hand are identical, namely those defined by the Borel cylinder sets, the weakly or strongly closed sets, and the weakly or strongly compact sets (see e.g. [7]). The functions $a(\omega)$ defined above are at least strongly continuous and therefore measurable with respect to this $\sigma$-algebra.

With a measure $d \mu_{\omega}$ it is possible to integrate a wider class of functions than those in $S(\mathscr{S})$. Moreover, a measure will not be uniquely determined by the functional it defines on $S(\mathscr{S})$. For this reason one would like to have a space $\mathscr{F}$ of functions on $\mathscr{S}_{R}^{\prime}$ containing $S(\mathscr{S})$, and a topology on $\mathscr{F}$ such that the measures we are interested in appear in a natural way as the dual space of $\mathscr{F}$. This suggests that $\mathscr{F}$ should be an algebra of functions over $\mathscr{S}_{R}^{\prime}$ which is at the same time a vector lattice, furnished with a kind of supremum topology. Since $\mathscr{F}$ shall contain $S(\mathscr{S})$ there is essentially a unique minimal choice satisfying these requirements:

2.1. Definition. (i) $\mathscr{F}$ consists of all functions $f: \mathscr{S}_{R}^{\prime} \rightarrow \mathbb{C}$ of the form

$$
f(\omega)=g\left(a_{1}(\omega), \ldots, a_{n}(\omega)\right)
$$

where $a_{i} \in S(\mathscr{S})$ and $g$ is a polynomially bounded continuous function on $\mathbb{C}^{n}$.

(ii) A topology $\hat{\tau}$ on $\mathscr{F}$ is defined by the collection of all seminorms of the form

$$
\|f\|_{F}=\sup _{\omega} \frac{|f(\omega)|}{F(\omega)}
$$

where $F$ is a function $\mathscr{S}_{R}^{\prime} \rightarrow[0, \infty]$ of the following type:

There is a seminorm $p$ on $S(\mathscr{S})$, continuous in the usual topology $\tau$, such that

$$
F(\omega)=F_{p}(\omega) \equiv \sup \{|a(\omega)| \mid p(a) \leqq 1\} .
$$


Remark. The restriction of this new topology $\hat{\tau}$ to $S(\mathscr{S})$ is coarser than the usual topology $\tau$. This follows from the bipolar theorem which says that for any seminorm $p$ we have

$$
p(a)=\sup _{T \in S(\mathscr{S})^{\prime}} \frac{|T(a)|}{p^{0}(T)} \quad \text { with } \quad p^{0}(T) \equiv \sup \{|T(a)| \mid p(a) \leqq 1\} .
$$

Since $F_{p}(\omega)=p^{0}\left(\chi_{\omega}\right)$ by definition, this implies $\|a\|_{F_{p}} \leqq p(a)$. In the next section we shall see that $\hat{\tau} \uparrow S(\mathscr{S})$ is strictly coarser than $\tau$.

The minimal choice of $\mathscr{F}$ has the following consequence:

2.2. Lemma. If $d \mu$ is a measure on $\mathscr{S}_{R}^{\prime}$ such that every $a \in S(\mathscr{S})$ is integrable, then also every $f \in \mathscr{F}$ is integrable w.r.t. $d \mu$.

Proof. Integrability w.r.t. a complex measure means the same as integrability w.r.t. the positive measure $d|\mu|$, and every $f \in \mathscr{F}$ is obviously measurable and dominated as a function on $\mathscr{S}_{R}^{\prime}$ by some $a \in S(\mathscr{S})$.

Our aim is now to prove

2.3. Theorem. For a linear functional $\hat{T}$ on $\mathscr{F}$ the following are equivalent:

(i) $\hat{T}$ is $\hat{\tau}$-continuous

(ii) there is a unique complex measure $d \mu$ on $\mathscr{S}_{R}^{\prime}$ such that every $f \in \mathscr{F}$ is integrable and

$$
\hat{T}(f)=\int f(\omega) d \mu_{\omega} .
$$

By the Hahn-Banach theorem and Lemma 2.2 this result has the following corollary:

2.4. Theorem. A linear functional $T$ defined on a subspace of $S(\mathscr{S})$ has a representation

$$
T(a)=\int a(\omega) d \mu_{\omega}
$$

with a complex measure $d \mu$ for which all $a \in S(\mathscr{S})$ are integrable if and only if $T$ is continuous in the restriction of $\hat{\tau}$ to this subspace.

Proof of Theorem 2.3. We show first that (ii) $\Rightarrow$ (i). Recall that a linear functional $T$ on $S(\mathscr{S})$ is called strongly positive if it is positive on all $a$ with $a(\omega) \geqq 0$ for all $\omega$. The cone of such positive $a$ 's contains (properly) the closed cone generated by all squares $a^{*} a$.

2.5. Lemma. Every strongly positive linear functional on $S(\mathscr{S})$ is $\tau$-continuous.

Proof. The assertion follows from the fact that the closed cone generated by all $a^{*} a$ is a complete strict $b$-cone in the bornological space $S(\mathscr{S})$, cf. [8], Theorem 5.5 , p. 228 and [9].

Remark. We note that the completeness of $S(\mathscr{S})$ is crucial for this lemma, for there certainly exist discontinuous characters on the incomplete tensor algebra.

From this we get immediately

2.6. Lemma. Let $d \mu_{\omega}$ be a complex measure on $\mathscr{S}_{R}^{\prime}$ such that every $a \in S(\mathscr{S})$ is 
integrable. Then the linear functional on $S(\mathscr{S})$

$$
T(a)=\int a(\omega) d \mu_{\omega}
$$

is $\tau$-continuous.

Proof. By the Hahn-decomposition we may write $d \mu_{\omega}$ as a linear combination of positive measures. But a positive measure defines a strongly positive functional, so Lemma 2.5 applies.

For the remaining part of (ii) $\Rightarrow$ (i) we now use the nuclear spectral theorem ([10], p. 83 or [11], Chapter I $\S 4.4$ ). As in the preceeding lemma we may restrict ourselves to a positive measure $d \mu_{\omega}$. Since $1 \in S(\mathscr{S})$ is integrable, $d \mu_{\omega}$ is a finite measure. From Lemma 2.6 and the fact that every weakly continuous representation of $S(\mathscr{S})$ is strongly continuous (cf. e.g. [1], Lemma 4.2) it follows that the canonical mapping of $S(\mathscr{S})$ into the Hilbert space $L_{2}\left(\mathscr{S}_{R}^{\prime}, d \mu_{\omega}\right)$ is continuous. By the nuclear spectral theorem there exists therefore a non-negative function $c(\cdot) \in L_{2}\left(\mathscr{S}_{R}^{\prime}, d \mu_{\omega}\right) \subset L_{1}\left(\mathscr{S}_{R}^{\prime}, d \mu_{\omega}\right)$ and a continuous seminorm $p$ on $S(\mathscr{S})$ such that $|a(\omega)| \leqq c(\omega) p(a)$ for all $a \in S(\mathscr{S}), \omega \in \mathscr{Y}_{R}^{\prime}$. This estimate implies that for the function $F_{p}$ of Definition 2.1 we have $F_{p}(\omega) \leqq c(\omega)$ and therefore

$$
\begin{aligned}
|\hat{T}(f)| & \leqq \int|f(\omega)| d \mu_{\omega} \leqq \sup _{\omega} \frac{|f(\omega)|}{c(\omega)} \cdot \int c\left(\omega^{\prime}\right) d \mu_{\omega^{\prime}} \\
& \leqq k \cdot\|f\|_{F_{p}} \text { with } k=\int c(\omega) d \mu_{\omega}<\infty .
\end{aligned}
$$

This completes the proof of (ii) $\Rightarrow$ (i).

To show the converse we note first that the real part $\mathscr{F}_{R}$ of $\mathscr{F}$ is a vector lattice in the natural order for functions, and the topology $\hat{\imath}$ is obviously compatible with the lattice structure in the sense that $|f| \leqq|g|$ implies $\|f\|_{F} \leqq\|g\|_{F}$. Hence, the topological dual $\mathscr{F}_{R}^{\prime}$ is also a vector lattice ([8], Theorem 7.4, p. 237) so every $\hat{T} \in \mathscr{F}^{\prime}$ can be written as $\hat{T}=\left(\hat{T}_{1}-\hat{T}_{2}\right)+i\left(\hat{T}_{3}-\hat{T}_{4}\right)$ with positive (and $\hat{\tau}$-continuous) functionals $\hat{T}_{i}$. The proof is therefore complete if we know that every positive functional on $\mathscr{F}$ is given by a measure. This may be shown in the same way as in [2] Section 3, but we shall here give a different proof more along the line of Theorem 4.3 in [1].

2.7. Theorem. The following are equivalent for a linear functional $\hat{T}$ on $\mathscr{F}$ :

(i) $\hat{T}$ is positive

(ii) There is a unique positive measure $d \mu$ on $\mathscr{S}_{R}^{\prime}$ such that

$$
\hat{T}(f)=\int f(\omega) d \mu_{\omega} \text { for all } f \in \mathscr{F} \text {. }
$$

Proof. Suppose $\hat{T}$ is positive and let $\hat{\pi}$ be the cyclic representation of $\mathscr{F}$ defined by $\hat{T}$ with cyclic vector $\Omega$ and domain $\hat{\mathscr{D}}=\hat{\pi}(\mathscr{F}) \Omega$. Let $\mathscr{B} C \mathscr{F}$ be the bounded functions in $\mathscr{F}$. By positivity of $\hat{T}$ one has $\|\hat{\pi}(b)\| \leqq \sup |b(\omega)|$ for all $b \in \mathscr{B}$, so the norm closure of $\hat{\pi}(\mathscr{B})$ is an abelian $C^{*}$-algebra $\hat{\mathscr{M}}$ of bounded operators commuting strongly with all $\hat{\pi}(f)$ 's. For any real function $f \in \mathscr{F}, f \pm i$ has an inverse in $\mathscr{B}$, so $\hat{\pi}(f) \pm i$ has an inverse in $\hat{\mathscr{M}}$. The operators $\hat{\pi}(f)$ for $f$ real are therefore essentially self-adjoint. By uniqueness of the functional calculus for self-adjoint operators, $\hat{\mathscr{M}}$ contains all bounded continuous functions of the $\hat{\pi}(f)$ 's. Since $\hat{\pi}(\mathscr{F})$ and therefore also $\hat{\mathscr{M}}$ has a cyclic vector we have that $\hat{\mathscr{M}}^{\prime \prime}$ is maximal abelian, so 
all the operators $\hat{\pi}(f)$ can be written as multiplication operators on $L_{2}\left(\Lambda, d v_{\lambda}\right)$, where $d v_{\lambda}$ is a measure on the spectrum $\Lambda$ of $\hat{M}$. From the nuclear spectral theorem one concludes as in [1], Theorem 4.3, that there is a mapping $\Lambda \ni \lambda \mapsto \omega_{\lambda} \in \mathscr{Y}_{R}^{\prime}$, such that for $a \in S(\mathscr{S}), \hat{\pi}(a)$ is represented by the function $\lambda \mapsto a\left(\omega_{\lambda}\right)$. Again by uniqueness of the functional calculus for self-adjoint operators this holds for all $f \in \mathscr{F}$, because every $\hat{\pi}(f)$ is a continuous function of some $\hat{\pi}\left(a_{1}\right), \ldots \hat{\pi}\left(a_{n}\right) ; a_{i} \in S(\mathscr{S})$. Hence

$$
\hat{T}(f)=\int_{\Lambda} f\left(\omega_{\lambda}\right) d v_{\lambda} .
$$

The measure on $\Lambda$ is then transported into a measure on $\mathscr{S}_{R}^{\prime}$ in the same way as in [1]. The uniqueness of the measure follows from the uniqueness of the decomposition of $\hat{\mathscr{M}}$.

\section{Restriction of the Topology $\hat{\tau}$ to the Tensor Algebra}

The seminorms \|\|$_{F}$ which define the topology $\hat{\tau}$ were tailored to fit the interpretation of $S(\mathscr{S})$ as an algebra of functions on $\mathscr{S}_{R}^{\prime}$. However $S(\mathscr{S})$ is also a graded algebra and this grading is in no way manifest in the structure of the seminorms \|\|$_{F}$. For applications one would like to have the topology $\hat{\tau}$ on $S(\mathscr{S})$ given by a class of $\tau$-continuous seminorms of the conventional form. This requires more detailed consideration than have been necessary so far, but once it has been achieved it will also become possible to identify $\hat{\tau}$ with other natural topologies on $S(\mathscr{S})$. This last aspect of the problem will be dealt with in the next section.

In the first step towards this goal we replace the infinite dimensional space $\mathscr{S}_{R}$ by $\mathbb{R}$ and consider the analogous problem for the symmetric tensor algebra over one-dimensional space. This is the same as the algebra $C[X]$ of complex polynomials of one (real) variable. The analogue of the topology $\tau$ is here the finest locally convex topology, which can be defined by the seminorms

$$
\|P\|_{\left\{c_{n}\right\}}=\sum_{n=0}^{N} c_{n}\left|\alpha_{n}\right|
$$

for $P(x)=\sum_{n=0}^{N} \alpha_{n} x^{n}$, where $\left\{c_{n}\right\}$ is an arbitrary sequence with $0 \leqq c_{n}<\infty$. The analogue of $\hat{\tau}$ is given by the seminorms

$$
\|P\|_{F}=\sup _{x \in \mathbb{R}} \frac{|P(x)|}{F(x)}
$$

where $F(x)=\sum_{n=0}^{\infty} d_{n}|x|^{n}$, with some constants $0<d_{n} \leqq \infty . F(x)$ is then a function which grows faster than any polynomial, so $\|P\|_{F}<\infty$ for all $P \in C[X]$.

3.1. Lemma. Both classes of seminorms, \|\|$_{\left\{c_{n}\right\}}$ resp. \|\|$_{F}$ define the same topology on $C[X]$.

Proof. The statement is essentially a consequence of a result of Boas [12] which asserts that every linear functional on $C[X]$ is a linear combination of positive functionals and therefore given by a complex measure on $\mathbb{R}$. In fact we need a slightly stronger version of this, which can easily be extracted from the 
proof in [12] (see also [13] statement 3): For any sequence $\left\{c_{n}\right\}$ there is a sequence $\left\{c_{n}^{\prime}\right\}$ such that any $T \in C[X]^{\prime}$ with $|T| \leqq\|\|_{\left\{c_{n}\right\}}$ can be written as $\left(T_{1}-T_{2}\right)+i\left(T_{3}-T_{4}\right)$ with positive functionals $T_{i}$ satisfying $\left|T_{i}\right| \leqq\|\|_{\left\{c_{n}^{\prime}\right\}}$. (This means that the positive cone in $C[X]$ is normal.) It follows that for any $T$ with $|T| \leqq\|\|_{\left\{c_{n}\right\}}$ there is a measure $d \mu_{x}$ on $\mathbb{R}$ such that

$$
T(P)=\int P(x) d \mu_{x}
$$

and $\int|x|^{n} d|\mu| \leqq 4 c_{n}^{\prime}$. With $d_{n}:=2^{-(n+1)} \cdot\left(4 c_{n}^{\prime}+1\right)^{-1}$ and $F(x)=\sum_{n=0}^{\infty} d_{n}|x|^{n}$ we get

$$
|T(P)| \leqq \int|P(x)| d|\mu| \leqq\|P\|_{F} \cdot \int F(x) d\left|\mu_{x}\right| \leqq\|P\|_{F} .
$$

This shows that \|\|$_{\left\{c_{n}\right\}} \leqq\|\|_{F}$. Conversely it is obvious that the seminorms \|\|$_{\left\{c_{n}\right\}}$ define a finer topology than the seminorms \|\|$_{F}$.

For the algebra $S(\mathscr{S})$ the situation is not as simple but the lemma can now be used to prove the following result.

3.2. Lemma. Let $a_{n}$ denote the components of $a \in S(\mathscr{S})$ in $S_{n}(\mathscr{S})$. For any $\hat{\tau}$ continuous seminorm \|\|$_{F}$ on $S(\mathscr{S})$ and any sequence $\left\{c_{n}\right\}$ with $0 \leqq c_{n}<\infty$ the seminorm

$$
a \mapsto \sum_{n} c_{n}\left\|a_{n}\right\|_{F}
$$

is also $\hat{\tau}$-continuous.

Proof. By the previous lemma there exists a function $G(x)=\sum_{n=0}^{\infty} d_{n}|x|^{n}$ with $d_{n}>0$ such that

$$
\sum_{n} 2^{n+1} c_{n}\left|\alpha_{n}\right| \leqq \sup _{x} \frac{\left|\sum_{n} \alpha_{n} x^{n}\right|}{G(x)}
$$

for all finite sequences $\left\{\alpha_{n}\right\}$. Hence,

$$
\begin{aligned}
\sum_{n} c_{n}\left\|a_{n}\right\|_{F} & \leqq \sup _{n} 2^{n+1} c_{n}\left\|a_{n}\right\|=\sup _{\omega} \sup _{n} 2^{n+1} \cdot c_{n} \frac{\left|a_{n}(\omega)\right|}{F(\omega)} \\
& \leqq \sup _{\omega} \sum_{n=0}^{\infty} 2^{n+1} \cdot c_{n} \frac{\left|a_{n}(\omega)\right|}{F(\omega)} \leqq \sup _{\omega} \sup _{x}\left|\sum_{n=0}^{\infty} \frac{a_{n}(\omega) x^{n}}{F(\omega) \cdot G(x)}\right| .
\end{aligned}
$$

From the Definition 2.1 we have $F(\omega)=F_{p}(\omega)=\sup \{|a(\omega)| \mid p(a) \leqq 1\}$ with some $\tau$-continuous norm $p$, which we may take of the form $p(a)=\sup _{n} p_{n}\left(a_{n}\right)$. Here $p_{n}$ is a continuous norm on $S_{n}(\mathscr{S})$ and it follows that

$$
F(\omega)=\sum_{n} F_{n}(\omega) \quad \text { with } \quad F_{n}(\omega)=\sup \left\{\left|a_{n}(\omega)\right| \mid p_{n}\left(a_{n}\right) \leqq 1\right\}
$$

Since $a_{n}(\omega \cdot x)=a_{n}(\omega) \cdot x^{n}$ we have also $F_{n}(\omega \cdot x)=F_{n}(\omega) \cdot|x|^{n}$ and $F(\omega) \cdot G(x) \geqq$ 
$\sum_{n} d_{n} F_{n}(\omega \cdot x)$. It follows that $\sum c_{n}\left\|a_{n}\right\|_{F}$ is dominated by the $\hat{\tau}$-norm $\|a\|_{F_{q}}$ with $q(a)=\sup _{n} d_{n}^{-1} p_{n}(a)$.

In order to carry the investigation further it is now necessary to specify the form of the functions $F$ which define the topology $\hat{\tau}$. Of course it is enough to describe a basis of functions, i.e. such that the corresponding seminorm form a basis for the topology $\hat{\tau}$. From the remark following Definition 2.1 we recall the notation $p^{0}$ for the dual Minkowski functional of a seminorm $p \cdot p^{0}$ is a norm on the linear space $\left\{T \mid p^{0}(T)<\infty\right\}$, which is dense in the dual space iff $p$ is a norm.

3.3. Lemma. The functions of either one of the following two types form a basis:

(i) $F(\omega)=\sum_{v=0}^{\infty} p_{v}^{0}(\omega)^{v}$

(ii) $F(\omega)=\prod_{v=1}^{\infty}\left(1+p_{v}^{0}(\omega)\right)$

where $\left\{p_{v}\right\}$ is any sequence of continuous seminorms on $\mathscr{S}$.

Proof. For the topology $\tau$ the seminorms of the form $p(a)=\sup _{n} p_{n} \otimes_{\varepsilon} \ldots \otimes_{\varepsilon} p_{n}\left(a_{n}\right)$ with continuous seminorm $p_{n}$ on $\mathscr{S}$ form a basis. (For the definition of the tensor products see e.g. [14], Section 7.1. Our notation is possibly not quite standard, the $\varepsilon$-product is in [14] denoted by $\varepsilon(U, V)$ and the $\pi$-product by $\pi(U, V)$.) The dual form of this is $p^{0}(T)=\sum_{v=0}^{\infty} p_{v}^{0} \otimes_{\pi} \cdots \otimes_{\pi} p_{v}^{0}\left(T_{v}\right)$. With $T=\chi_{\omega}$ we get (i).

It is obvious that the second form of $F$ dominates the first one. In showing the converse, we may without restriction assume that $p_{v} \leqq p_{v+1}$, which implies $p_{v}^{0}(\omega) \geqq p_{v+1}^{0}(\omega)$. Then

$$
\sum_{v=0}^{\infty} p_{v}^{0}(\omega)^{v} \geqq \sum_{\mu=1}^{\infty} \frac{1}{2^{\mu}} \sum_{v=0}^{2^{\mu}} p_{v}^{0}(\omega)^{\nu} \geqq \sum_{\mu=1}^{\infty} \frac{1}{2^{\mu}} \sum_{v=0}^{2^{\mu}} p_{2^{\mu}}^{0}(\omega)^{\nu} \geqq \sum_{\mu=1}^{\infty} \frac{1}{2^{\mu}}\left(1+\varepsilon_{2^{\mu}} p_{2^{\mu}}^{0}(\omega)\right)^{2^{\mu}}
$$

where $\varepsilon_{n}>0$ is sufficiently small. By an elementary convexity inequality this is larger than

$$
\prod_{\mu=1}^{\infty}\left(1+\varepsilon_{2^{\mu}} p_{2^{\mu}}^{0}(\omega)\right)=\prod_{\mu=0}^{\infty}\left(1+q_{\mu}^{0}(\omega)\right) \quad \text { with } \quad q_{\mu} \equiv \varepsilon_{2^{\mu}}^{-1} p_{2^{\mu}} .
$$

As next we show that when we restrict \|\|$_{F}$ to $S_{n}(\mathscr{S})$ only the first $n$ factors in the product in Lemma 3.3. (ii) are relevant.

3.4. Lemma. For every sequence $\left\{p_{v}\right\}$ of continuous seminorms on $\mathscr{S}$ there is a $\hat{\tau}$-norm \|\|$_{G}$ such that for all $a_{n} \in S_{n}(\mathscr{S})$

$$
\sup _{\omega} \frac{\left|a_{n}(\omega)\right|}{p_{1}^{0}(\omega) \ldots p_{n}^{0}(\omega)} \leqq\left\|a_{n}\right\|_{G}
$$


Proof. We may assume that $p_{v}^{0} \geqq p_{v+1}^{0}$ and we shall define $G(\omega)=\prod_{v=1}^{\infty}\left(1+q_{v}^{0}(\omega)\right)$ with $q_{v}^{0}(\omega)=\varepsilon_{v} p_{v}^{0}$ where $\varepsilon_{v}>0$ and $\varepsilon_{v} \geqq \varepsilon_{v+1}$. In order to determine how small $\varepsilon_{v}$ must be we compute

$$
\begin{aligned}
\sup _{\omega} \frac{\left|a_{n}(\omega)\right|}{\prod_{\nu=1}^{\infty}\left(1+q_{\nu}^{0}(\omega)\right)} & \geqq \sup _{\omega} \frac{\left|a_{n}\left(\frac{\omega}{q_{n}^{0}(\omega)}\right)\right|}{\prod_{\nu=1}^{\infty}\left(1+q_{v}^{0}\left(\frac{\omega}{q_{n}^{0}(\omega)}\right)\right)} \\
& =\sup _{\omega} \frac{\left|a_{n}(\omega)\right|}{q_{n}^{0}(\omega)^{n} \prod_{\nu=1}^{\infty}\left(1+\frac{q_{v}^{0}(\omega)}{q_{n}^{0}(\omega)}\right)} \\
& =\sup _{\omega} \frac{\left|a_{n}(\omega)\right|}{\prod_{\nu=1}^{n}\left(q_{v}^{0}(\omega)+q_{n}^{0}(\omega)\right) \prod_{\nu=n+1}^{\infty}\left(1+\frac{q_{v}^{0}(\omega)}{q_{n}^{0}(\omega)}\right)} \\
& \geqq \sup _{\omega} \frac{\left|a_{n}(\omega)\right|}{p_{1}^{0}(\omega) \ldots p_{n}^{0}(\omega)} \cdot\left(2^{-n} \cdot \prod_{\nu=1}^{n} \varepsilon_{v}^{-1} \cdot \prod_{\nu=n+1}^{\infty}\left(1+\frac{\varepsilon_{v}}{\varepsilon_{n}}\right)^{-1}\right) .
\end{aligned}
$$

The last factor is $\geqq 1$ e.g. for $\varepsilon_{v}=2^{-(v+2)}$.

In order to relate the left hand side in Lemma 3.4 to more conventional seminorms we must first say a few words about how seminorms on the usual tensor power $\mathscr{S}_{n}=\mathscr{S}\left(\mathbb{R}^{d \cdot n}\right)$ resp. the usual tensor algebra $\mathscr{S}=\bigoplus_{n=0}^{\infty} \mathscr{S}_{n}$ induce seminorms on $S_{n}(\mathscr{S})$ resp. $S(\mathscr{S})$. Since we are interested in symmetric functionals $T=\left(T_{0}\right.$, $\left.T_{1}, \ldots\right) \in S(\mathscr{S})^{\prime}$ we may also look at $T$ as an element of $\mathscr{S}^{\prime}$ annihilating the closed two-sided ideal $\mathscr{I} \subset \mathscr{S}$ generated by the commutators. From this point of view it is natural to look at $S(\mathscr{S})$ as the quotient algebra $\mathscr{S} / \mathscr{I}$ and correspondingly identify $S_{n}(\mathscr{S})$ with $\mathscr{S}_{n} / \mathscr{I}_{n}$ where $\mathscr{I}_{n}=\mathscr{I}_{\cap} \mathscr{S}_{n}$. In accordance with this interpretation of $S_{n}(\mathscr{S})$ and $S(\mathscr{S})$ we make the following definition:

3.5. Definition. If $p$ is a seminorm on $\mathscr{S}_{n}$ we define its symmetrization as the quotient seminorm

$$
(p)_{s}(x)=\operatorname{Inf}_{y \in \mathscr{I}_{n}} p(x+y)
$$

and analogously for seminorms on $\underline{\mathscr{S}}$.

With this notation ${ }^{1}$ we get

3.6. Theorem. The topology $\hat{\tau}$ on $S(\mathscr{S})$ is given by the collection of the following seminorms

$$
p(a)=\sum_{n=0}^{\infty}\left(p_{1} \otimes_{\pi} \ldots \otimes_{\pi} p_{n}\right)_{s}\left(a_{n}\right)
$$

where $\left\{p_{v}\right\}$ is a sequence of continuous seminorms on $\mathscr{S}$.

The notation is perhaps a little ambiguous. As defined above, $(p)_{s}$ is a seminorm on $\mathscr{S}_{n}$. However, since it is constant on every equivalence class $x+\mathscr{I}_{n}$ it corresponds uniquely to a seminorm on $S_{n}(\mathscr{S})$. 
Proof. To begin with, nuclearity of $\mathscr{S}$ allows us to replace the $\pi$-products $p_{1} \otimes_{\pi} \ldots \otimes_{\pi} p_{n}$ by the (strictly smaller) $\varepsilon$-products $\left(p_{1} \otimes_{\varepsilon} \ldots \otimes_{\varepsilon} p_{n}\right)$ without changing the topology. According to Lemma 3.4 it is therefore enough to show that

$$
\left(p_{1} \otimes_{\varepsilon} \ldots \otimes_{\varepsilon} p_{n}\right)_{s}(a) \leqq c_{n} \cdot \sup _{\omega} \frac{|\omega \otimes \ldots \otimes \omega(a)|}{p_{1}^{0}(\omega) \ldots p_{n}^{0}(\omega)}
$$

with some constant $c_{n}$ independent of $a \in \mathscr{S}_{n}$.

For this task we shall make use of the Hermite expansion of elements in $\mathscr{S}$. Every $a \in \mathscr{S}$ can be written as $a=\sum_{v=1}^{\infty} \alpha_{v} e_{v}$ where $\left\{\alpha_{v}\right\}$ is a sequence of rapid decrease and the $e_{v}$ 's are the Hermite functions which form as basis for $\mathscr{S}$. Similarly, every $a \in \mathscr{S}_{n}$ has the expansion

$$
a=\sum_{v_{1} \ldots v_{n}} \alpha_{v_{1} \ldots v_{n}} e_{v_{1}} \otimes \ldots \otimes e_{v_{n}}
$$

where $\left\{\alpha_{v_{1} \ldots v_{n}}\right\}$ decreases strongly in all indices. It is enough to prove (1) for $p_{v}$ 's taken from a basis of seminorms on $\mathscr{S}$ and a convenient basis for our purpose is provided by the seminorms

$$
p^{(k)}(a)=\sup _{v}\left|v^{k} \alpha_{v}\right|=\sup _{v}\left|\left\langle v^{k} e_{v}^{*}, a\right\rangle\right|
$$

where $k$ is an integer and $e_{v}^{*} \in \mathscr{S}^{\prime}$ denotes the dual basis of $e_{v}$, defined by $\left\langle e_{v}^{*}, e_{\mu}\right\rangle=$ $\delta_{\mu v}$. If $p_{1}, \ldots, p_{n}$ are seminorms of this form, corresponding to the exponents $k_{1}, \ldots, k_{n}$, their $\varepsilon$-product is given by

$$
p_{1} \otimes_{\varepsilon} \ldots \otimes_{\varepsilon} p_{n}(a)=\sup _{v_{1} \ldots v_{n}}\left|v_{1}^{k}{ }^{1} \ldots v_{n}^{k_{n}}\left\langle e_{v_{1}}^{*} \otimes \ldots \otimes e_{v_{n}}^{*}, a\right\rangle\right| .
$$

We must as next take the symmetrization of $p_{1} \otimes_{\varepsilon} \ldots \otimes_{\varepsilon} p_{n}$ into account. Without restriction one may suppose that $k_{1} \leqq \ldots \leqq k_{n}$. For $a \in \mathscr{S}_{n}$ as in (2), we define

$$
\bar{a}=\sum_{v_{1} \geqq \ldots \geqq v_{n}} \bar{\alpha}_{v_{1} \ldots v_{n}} e_{v_{1}} \otimes \ldots \otimes e_{v_{n}}
$$

where $\bar{\alpha}_{v_{1} \ldots v_{n}}$ is the sum over all $\alpha_{\mu_{1} \ldots \mu_{n}}$ such that $\left(\mu_{1}, \ldots, \mu_{n}\right)$ is a permutation of $\left(v_{1}, \ldots, v_{n}\right)$. Since obviously $\bar{a} \in a+\mathscr{I}_{n}$ it follows by Definition 3.5 and by (3) that

$$
\left(p_{1} \otimes_{\varepsilon} \ldots \otimes_{\varepsilon} p_{n}\right)_{s}(a) \leqq p_{1} \otimes_{\varepsilon} \ldots \otimes_{\varepsilon} p_{n}(\bar{a})=\left|\bar{v}_{1}^{k_{1}} \ldots \bar{v}_{n}^{k_{n}}\left\langle e_{\bar{v}_{1}}^{*} \otimes \ldots \otimes e_{\bar{v}_{n}}^{*}, \bar{a}\right\rangle\right|
$$

for some $\bar{v}_{1} \geqq \ldots \geqq \bar{v}_{n}$. We write this last expression more compactly as $\left|\left\langle\omega_{1} \otimes \ldots \otimes \omega_{n}, \bar{a}\right\rangle\right|$ with $\omega_{i}=\bar{v}_{i}^{k_{i}} e_{v_{1}}^{*}$.

By a general polarization identity we may for any constants $\lambda_{1}, \ldots, \lambda_{n}$ write the symmetric expression

$$
\lambda_{1} \ldots \lambda_{n} \sum_{\text {Perm. } \pi} \omega_{\pi 1} \otimes \ldots \otimes \omega_{\pi n}
$$

as a linear combination of terms of the form $\left(\lambda_{i_{1}} \omega_{i_{1}}+\ldots+\lambda_{i_{l}} \omega_{i_{l}}\right)^{\otimes n}$. Since $\omega_{\pi 1} \otimes \ldots \otimes \omega_{\pi n}$ applied to $\bar{a}$ is either the same as $\left\langle\omega_{1} \otimes \ldots \otimes \omega_{n}, a\right\rangle$ or zero (in the 
definition of $\bar{a}$ the sum is taken over $\left.v_{1} \geqq \ldots \geqq v_{n}\right)$, this means that for some $i_{1} \ldots i_{l}$ (depending on $\lambda_{1}, \ldots, \lambda_{n}$ ) we have

$$
\left|\lambda_{1} \ldots \lambda_{n}\left\langle\omega_{1} \otimes \ldots \otimes \omega_{n}, \bar{a}\right\rangle\right| \leqq c_{n}^{\prime}|\langle\omega \otimes \ldots \otimes \omega, \bar{a}\rangle|
$$

with $\omega=\lambda_{i_{1}} \omega_{i_{1}}+\ldots+\lambda_{i_{l}} \omega_{i_{l}}$ and $c_{n}^{\prime}$ a constant depending only on $n$. Moreover, for any $i_{1}, \ldots, i_{l}$ and $\lambda_{j} \geqq 0$ we have $p_{i}^{0}(\omega) \leqq n \cdot \max \lambda_{j} p_{i}^{0}\left(\omega_{j}\right)$. It follows that if we can choose $\lambda_{i}$ such that $\lambda_{j} p_{i}^{0}\left(\omega_{j}\right) \leqq \lambda_{i}$ for all $i, j$ then we have

$$
\left|\left\langle\omega_{1} \otimes \ldots \otimes \omega_{n}, \bar{a}\right\rangle\right| \leqq c_{n}^{\prime} \cdot n^{n} \frac{|\langle\omega \otimes \ldots \otimes \omega, \bar{a}\rangle|}{p_{1}^{0}(\omega) \ldots p_{n}^{0}(\omega)}
$$

which implies (1) and the proof will be complete. So let us show that such a choice of $\lambda_{i}$ is possible. We have $p_{i}^{0}\left(\omega_{j}\right)=\bar{v}_{j}^{k_{j}-k_{2}}$ so we must show that $\bar{v}_{j}^{k_{j}-k_{i}} \leqq \frac{\lambda_{i}}{\lambda_{j}}$ for all $i, j$ which means the same as

$$
\bar{v}_{j}^{k_{j}-k_{\imath}} \leqq \frac{\lambda_{i}}{\lambda_{j}} \leqq \bar{v}_{i}^{k_{j}-k_{\imath}}
$$

for $i \leqq j$. We now define $\lambda_{i}$ by induction such that this holds for all $i$ and $j=i+1$. This can be done because $\bar{v}_{i+1} \leqq \bar{v}_{i}$ and $k_{i+1} \geqq k_{i}$. For arbitrary $j \geqq i$ we then write

$$
\frac{\lambda_{i}}{\lambda_{j}}=\frac{\lambda_{i}}{\lambda_{i+1}} \cdots \frac{\lambda_{j-1}}{\lambda_{j}}
$$

and obtain

$$
\frac{\lambda_{i}}{\lambda_{j}} \leqq \bar{v}_{i}^{k_{2+1}-k_{i}} \ldots \bar{v}_{j-1}^{k_{j}-k_{j-1}} \leqq \bar{v}_{i}^{k_{i+1}-k_{\imath}} \ldots \bar{v}_{i}^{k_{j}-k_{j-1}}=\bar{v}_{i}^{k_{j}-k_{\imath}}
$$

and in the same way the other inequality.

Remark. In the proof of this theorem we have for technical reasons used the fact that $\mathscr{S}$ has a basis. Although there are other test function spaces, e.g. $\mathscr{D}$, which do not have this property it is easily seen that the proof goes through for spaces which are the inductive limit of spaces with basis. This is the case for all the usual nuclear test function spaces. At present, however, it is not clear whether the theorem holds for all nuclear spaces.

Combining this result with Theorem 2.4 we get

Theorem 3.7. Let $T=\left(T_{0}, T_{1}, \ldots\right)$ be a sequence of tempered distribution $T_{n} \in \mathscr{S}\left(\mathbb{R}^{d \cdot n}\right)^{\prime}$ with

$$
T_{n}\left(f_{1} \otimes \ldots \otimes f_{n}\right)=T_{n}\left(f_{\pi 1} \otimes \ldots \otimes f_{\pi n}\right)
$$

for all permutations $\pi$ and $f_{i} \in \mathscr{S}\left(\mathbb{R}^{d}\right)$. Then the following conditions are equivalent.

(i) There are continuous seminorms $p_{v}$ on $\mathscr{S}\left(\mathbb{R}^{d}\right)$ such that

$$
\left|T_{n}\left(f_{1} \otimes \ldots \otimes f_{n}\right)\right| \leqq p_{1}\left(f_{1}\right) \ldots p_{n}\left(f_{n}\right)
$$

for all $f_{i} \in \mathscr{S}$. 
(ii) There is a complex measure $d \mu$ on $\mathscr{S}_{R}^{\prime}$ such that

$$
T_{n}=\int \underbrace{\omega \otimes \ldots \otimes \omega}_{n \text {-factors }} d \mu_{\omega}
$$

as a weak integral in $\mathscr{S}_{n}^{\prime}$.

Proof. The estimate (i) and the symmetry of $T_{n}$ implies $\left|T_{n}\right| \leqq\left(p_{1} \otimes_{\pi} \ldots \otimes_{\pi} p_{n}\right)_{s}$.

\section{Other Interpretations of the Topology $\hat{\tau}$}

The main objective of this section is to show that the topology $\hat{\tau}$ on $S(\mathscr{S})$ can be characterized by a property which at the first glance is independent of the requirements which motivated the introduction of $\hat{\tau}$, but has to do with the continuity of the product on the algebra $S(\mathscr{S})$. At the same time we state some slight refinements of the results in Section 2. The essential property of $\hat{\tau}$ is that the dual space of $S(\mathscr{S})[\hat{\tau}]$ is generated by the strongly positive functionals, i.e. those which are positive on $S(\mathscr{S})^{+}:=\{a \mid a(\omega) \geqq 0$ for all $\omega\}$. A strengthened form of this is the normality of the cone $S(\mathscr{S})^{+}$which implies that a $\hat{\tau}$-equicontinuous set is a linear combination of $\hat{\tau}$-equicontinuous sets of strongly positive functionals. In $S(\mathscr{S})$ we may also consider the algebraic cone $S^{+}(\mathscr{S})=c l\left\{\sum_{i} a_{i}^{*} a_{i} \mid a_{i} \in S(\mathscr{S})\right\}$. The dual cone of $S^{+}(\mathscr{S})$ consists of the positive functionals. It is well known that there are positive functionals which are not strongly positive, but surprisingly enough, the equivalence of (iii) and (iv) in the theorem below means in particular that every positive functional is a difference of two strongly positive ones.

4.1. Theorem. On $S(\mathscr{S})$ the following topologies coincide:

(i) The topology $\hat{\tau}$.

(ii) The strongest locally convex topology $\tau_{m}$, coarser than $\tau$, such that the multiplication $S(\mathscr{S})\left[\tau_{m}\right] \times S(\mathscr{S})\left[\tau_{m}\right] \rightarrow S(\mathscr{S})\left[\tau_{m}\right]$ is jointly continuous.

(iii) The strongest topology such that $S(\mathscr{S})^{+}$is normal.

(iv) The strongest topology such that $S^{+}(\mathscr{S})$ is normal.

Proof. Let $\tau_{3}$ and $\tau_{4}$ denote the topologies defined in (iii) and (iv). We show first the equivalence of $\hat{\tau}, \tau_{3}$, and $\tau_{4}$. To begin with, $S(\mathscr{S})^{+}$is normal for $\hat{\tau}$ because the norms \|\|$_{F}$ have the property that $0 \leqq a \leqq b$ implies $\|a\|_{F} \leqq\|b\|_{F}$. This means that $\tau_{3} \succ \hat{\tau}$ and it is obvious that $\tau_{4} \succ \tau_{3}$ because $S^{+}(\mathscr{S}) \subset S(\mathscr{S})^{+}$. The dual space of $S(\mathscr{S})\left[\tau_{4}\right]$ is precisely the linear hull of the positive functionals and from the proof of Lemma 2.5 we know that positive functionals are $\tau$-continuous. Since $\tau$ is a Mackey topology it follows that $\tau \succ \tau_{4}$. Now $S^{+}(\mathscr{S})$ is normal for $\tau_{4}$ so it remains to show that a $\tau$-equicontinuous set of positive functionals is $\hat{\tau}$-equicontinuous. This follows from Theorem 3.6 and the Cauchy-Schwarz inequality:

If $T=\left(T_{0}, T_{1}, \ldots\right) \in S(\mathscr{S})[\tau]^{\prime}$ is a positive functional, we get by repeated application of the Cauchy-Schwarz inequality and the symmetry under permutations the estimate

$$
\left|T_{n}\left(f_{1} \otimes \ldots \otimes f_{n}\right)\right| \leqq \prod_{v=1}^{n} T_{2^{v}}\left(\left(f_{v}^{*}\right)^{\otimes 2^{v-1}} \otimes f_{v}^{\otimes 2^{v-1}}\right)^{2^{-v}}
$$


If $T$ belongs to an equicontinuous set defined by a $\tau$-seminorm

$$
p(a)=\sum_{v=0}^{\infty}\left(p_{\nu} \otimes_{\pi} \ldots \otimes_{\pi} p_{v}\right)\left(a_{n}\right)
$$

where $\left\{p_{v}\right\}$ is a sequence of seminorms on $\mathscr{S}$ with $p_{v}(f)=p_{v}\left(f^{*}\right)$, we have therefore also

$$
\left|T_{n}\left(f_{1} \otimes \ldots \otimes f_{n}\right)\right| \leqq q_{1}\left(f_{1}\right) \ldots q_{n}\left(f_{n}\right)
$$

with $q_{v}=p_{2^{v}}$. By Theorem 3.6 this shows that $T$ belongs to the $\hat{\tau}$-equicontinuous set defined by the seminorm

$$
q(a)=\sum_{n=0}^{\infty}\left(q_{1} \otimes_{\pi} \ldots \otimes_{\pi} q_{n}\right)_{s}\left(a_{n}\right) .
$$

This completes the proof of $\hat{\tau}=\tau_{3}=\tau_{4}$. For the equivalence of (i) and (ii) we show first that the multiplication is in fact continuous if $S(\mathscr{S})$ is equipped with the topology $\hat{\tau}$. That this is not true for the topology $\tau$ is e.g. shown in Lemma 5 of [15]. The continuity of the product is easily seen if we take $F(\omega)=\prod_{v=1}^{\infty}\left(1+p_{v}^{0}(\omega)\right)$ as in Lemma 3.3 with $p_{v}^{0} \geqq p_{v+1}^{0}$. Defining $G(\omega)=\prod_{v=1}^{\infty}\left(1+p_{v+1}^{0}(\omega)\right)$ we have $F(\omega) \geqq$ $\prod_{v=1}^{\infty}\left(1+p_{v+1}^{0}(\omega)\right)^{2}=G(\omega)^{2}$ so
$\quad\|a \cdot b\|_{F} \leqq\|a\|_{G} \cdot\|b\|_{G}$ for all $a, b \in S(\mathscr{S})$.

In order to verify that $\hat{\tau}$ is also the finest topology with a jointly continuous product we must have a more explicit description of this latter topology. Since this can be done for quite arbitrary algebras we formulate it as a separate result:

4.2. Proposition. Let $\mathfrak{U}$ be an algebra (not necessarily commutative), let $\mathfrak{b} \subset \mathfrak{U}$ be a linear space of algebraic generators for $\mathfrak{U}$ and let $t$ be any locally convex topology on $\mathbf{6}$. Among all locally convex topologies on $\mathfrak{A}$ having the properties

(a) the multiplication on $\mathfrak{A}$ is jointly continuous;

(b) the restriction to $(5$ is weaker than $t$;

there exists a finest one $t_{m}$ and a basis of neighbourhoods of 0 for $t_{m}$ is given by sets of the forms

$$
\mathfrak{U}=\text { absolutely convex hull } \bigcup_{n, \pi} U_{\pi 1} \ldots U_{\pi n}
$$

where the union is over all $n=1,2 \ldots$ and permutation $\pi$ and the sequence $\left\{U_{i}\right\}$ is taken from some basis of 0-neighbourhoods for $t$.

Proof. The existence of a finest topology $t_{m}$ with (a) and (b) follows immediately from Zorn's lemma. Let $\tilde{t}_{m}$ be the topology defined by the $\mathfrak{H}$ 's as in (4). Because $\mathfrak{5}$ generates $\mathfrak{A}$, the $\mathfrak{U}$ 's are absorbing and thus qualify as neighbourhoods of 0 . We show first that $\tilde{t}_{m}$ satisfies (a) and (b). The second condition is clear, because $\mathfrak{U}$ contains $U_{1}$ which is by assumption a $t$-neighbourhood in $\mathfrak{b}$. It is no restriction to assume that the sequence $U_{i}$ defining $\mathfrak{U}$ satisfies $U_{i+1} \subset U_{i}$. Define $V_{i}=U_{2 i}$ 
and $\mathfrak{B}=$ a.c.h. $\bigcup_{n, \pi} V_{\pi 1} \ldots V_{\pi n}$. We claim that $\mathfrak{B} \cdot \mathfrak{B} \subset \mathfrak{U}$. To see this, consider any two permutations and $\pi \in P_{n}$ and $\pi^{\prime} \in P_{m}$ and assume for sake of definiteness that $n \leqq m$. We then define a permutation $\pi^{\prime \prime} \in P_{n+m}$ in the following way:

$$
\begin{aligned}
& \pi^{\prime \prime}(i)=2 \pi(i)-1 \quad \text { for } i=1, \ldots, n \\
& \pi^{\prime \prime}(n+i)=\left\{\begin{array}{lll}
2 \pi^{\prime}(i) & \text { for } & \pi^{\prime}(i) \leqq n \\
n+\pi^{\prime}(i)=2 \pi^{\prime}(i)-\left(\pi^{\prime}(i)-n\right) & \text { for } & \pi^{\prime}(i)>n
\end{array}\right.
\end{aligned}
$$

This permutation satisfies $2 \pi(i) \geqq \pi^{\prime \prime}(i)$ for $i=1, \ldots, n$ and $2 \pi^{\prime}(i) \geqq \pi^{\prime \prime}(n+i)$ for $i=1, \ldots, m$.

Hence,

$$
\begin{aligned}
\left(V_{\pi 1} \ldots V_{\pi n}\right) \cdot\left(V_{\pi^{\prime} 1} \ldots V_{\pi^{\prime} m}\right)= & U_{2 \pi 1} \ldots U_{2 \pi 1} \cdot U_{2 \pi^{\prime} 1} \ldots U_{2 \pi^{\prime} m} \\
& \subset U_{\pi^{\prime \prime} 1} \ldots U_{\pi^{\prime \prime} n} \cdot U_{\pi^{\prime \prime}(n+1)} \ldots U_{\pi^{\prime \prime}(n+m)} .
\end{aligned}
$$

Taking the absolutely convex hull of the union over $\pi, \pi^{\prime}, n$, and $m$ yields $\mathfrak{B} \cdot \mathfrak{B} \subset \mathfrak{U}$, so $t_{m}$ is finer than $\tilde{t}_{m}$. For the converse, suppose $\mathfrak{U} \equiv \mathfrak{U}_{0}$ is any absolutely convex $t_{m}$-neighbourhood of 0 . Because of (a) there are $t_{m}$-neighbourhoods $\mathfrak{U}_{i}$ for $i=1,2 \ldots$ such that $\mathfrak{U}_{i+1} \cdot \mathfrak{U}_{i+1} \subset \mathfrak{U}_{i}$ for $i=0,1,2 \ldots$ and we may furthermore choose $\mathfrak{U}_{i}$ such that $\mathfrak{U}_{i+1} \subset \mathfrak{U}_{i}$. Because of (b), $U_{i} \equiv \mathfrak{U}_{i} \cap \mathfrak{b}$ is a $t$-neighbourhood of 0 . Now define $\tilde{\mathfrak{U}}_{i}=\mathfrak{U}_{2 i}$ and $\tilde{U}_{i}=U_{2 i}$. We claim that $\tilde{U}_{\pi 1} \ldots \tilde{U}_{\pi n} \subset \mathfrak{U}$ for all $n$ and $\pi \in P_{n}$. To see this, we prove by induction the following statement:

If $i_{1}, \ldots, i_{m}$ are numbers with $i_{r} \geqq 2$ and $\min _{r \neq s}\left|i_{r}-i_{s}\right| \geqq 2$, then $U_{i_{1}} \ldots U_{i_{n}} \subset \mathfrak{U}_{\alpha-2}$, where $\alpha=\min \left\{i_{1}, \ldots, i_{m}\right\}$. For $m=2$ the statement is trivial, so suppose it has been proven for $m \leqq n-1$. If $\min \left\{i_{1}, \ldots i_{n}\right\}=i_{r}$ we have then

$$
U_{i_{1}} \ldots U_{i_{r-1}} \subset \mathfrak{U}_{i_{r}} \text { and } U_{i_{r+1}} \ldots U_{i_{n}} \subset \mathfrak{U}_{i_{r}} \text {. }
$$

(If $r=1$ or $n$ put $U_{i_{0}}=U_{i_{n+1}}=\phi$.) Therefore,

$$
U_{i_{1}} \ldots U_{i_{n}} \subset U_{i_{r}} \cdot U_{i_{r}} \cdot U_{i_{r}} \subset \mathfrak{U}_{i_{r}-2}
$$

so the assertion holds for $m=n$. Since $\tilde{U}_{\pi i}=U_{2 \pi i}$ this shows that for all $\pi$ and $n$ $\tilde{U}_{\pi 1} \ldots \tilde{U}_{\pi n} \subset \mathfrak{U}$, so $\mathfrak{U}$ is a $\tilde{t}_{m}$-neighbourhood of 0 .

With this result we can now complete the proof of Theorem 4.1: For a commutative algebra the expression for $\mathfrak{U}$ simplifies to $\mathfrak{U}=\bigcup_{n=1}^{\infty} U_{1} \ldots U_{n}$. Taking $\mathfrak{U}=S(\mathscr{S})$ and $\mathfrak{b}=\mathbb{C} \oplus \mathscr{S}$ the seminorm corresponding to $\mathfrak{U}$ is

$$
p=\sum_{n=0}^{\infty}\left(p_{1} \otimes_{\pi} \ldots \otimes_{\pi} p_{n}\right)_{s}
$$

where $p_{v}$ is the seminorm defined by $U_{v}$. This is exactly the same expression as in Theorem 3.6.

Remarks. (1) The topologies $\tau_{m}$ and $\hat{\tau}$ are in a similar relation to one another as the original norm and the enveloping $C^{*}$-norm for a Banach algebra. The 
equivalence of these two topologies is therefore not to be expected in general. The essential property is nuclearity of $\mathscr{S}$ which entered crucially in Theorem 3.6.

(2) The analogue of Theorem 4.1 for the usual (non commutative) tensor algebra $\mathscr{S}$ does not hold. In this case $\hat{\tau}$ is replaced by a stronger topology, cf. [15] Theorem 4. It can be shown by an example that there exist symmetric functionals on $\mathscr{S}$ which can be written as a difference of two positive functionals but not as a difference of two positive and symmetric ones.

For completeness sake we conclude this section by mentioning some additional properties of $\hat{\tau}$ which may be proved in the same way as Theorem 6 in [15].

4.3. Proposition. $S(\mathscr{S})$ equipped with the topology $\hat{\tau}$ is nuclear and complete, but neither bornological nor barrelled.

\section{Consequences for the Wightman Functions}

The Wightman functions at Schwinger Points $\mathbb{S}_{n}$ are defined by analytic continuation from the usual Wightman functions $\mathscr{W}_{n}$ as $\mathfrak{S}\left(x_{1}, \ldots, x_{n}\right)=\mathscr{W}_{n}\left(i x_{1}^{0}, \boldsymbol{x}_{1}, \ldots, i x_{n}^{0}\right.$, $\left.\boldsymbol{x}_{n}\right)$, where $x^{0}, \boldsymbol{x}$ denote the time- resp. space coordinates of $x \in \mathbb{R}^{d}$. $\mathfrak{\Xi}_{n}$ is defined and real analytic at all points $\left(x_{1}, \ldots, x_{n}\right)$ with $x_{i} \neq x_{j}$ for all $i, j$. Moreover, the growth properties of $\mathfrak{S}_{n}$ in a neighbourhood of the singularities and at infinity are such [16] that $\mathfrak{S}_{n}\left(x_{1} \ldots x_{n}\right)$ may be integrated with test functions in $\mathscr{S}_{n}$ having a zero of infinite order on the hyperplanes $x_{i}=x_{j}$. In this way the sequence $\left\{\Theta_{n}\right\}$ defines a linear functional on a subspace of the tensor algebra. The continuity requirement of Theorem 3.6 can now be translated into a condition on the behaviour of $\mathfrak{S}_{n}$ as a function and the result is:

Theorem 5.1. Let $\mathfrak{\Xi}_{n}\left(x_{1} \ldots x_{n}\right) n=0,1,2 \ldots$ be the Wightman functions at Schwinger points for a field theory. A necessary and sufficient condition for the existence of a complex measure $d \mu$ on $\mathscr{S}_{R}^{\prime}$ such that

$$
\int_{\mathbb{R}^{d \cdot n}} \Xi_{n}\left(x_{1}, \ldots, x_{n}\right) f\left(x_{1}, \ldots, x_{n}\right) d x=\int_{\mathscr{S}_{R}^{\prime}} \omega \otimes \ldots \otimes \omega(f) d \mu_{\omega}
$$

for all $n$ and all test functions $f$ which have a zero of infinite order at coinciding points is the following:

There are constants $c_{n}, k_{n}$, and $l_{n}$ with

$$
\left|\Xi_{n}\left(x_{1}, \ldots, x_{n}\right)\right| \leqq c_{n} \cdot \sum_{v=2}^{n} \sum_{i_{1}<\ldots<i_{v}}\left\{d\left(x_{i_{1}} \ldots x_{i_{v}}\right)^{-k v}+r\left(x_{i_{1}} \ldots x_{i_{v}}\right)^{l v}\right\}
$$

where

$$
\begin{aligned}
& d\left(y_{1}, \ldots, y_{m}\right)=\max _{i, j}\left|y_{i}-y_{j}\right| \\
& r\left(y_{1}, \ldots, y_{m}\right)=\min _{i \neq j}\left|y_{i}-y_{j}\right| .
\end{aligned}
$$

The right-hand side of (5) then defines an extension of $\mathfrak{S}=\left(1, \mathfrak{S}_{1}, \mathfrak{S}_{2}, \ldots\right)$ to the whole of $S(\mathscr{S})$.

Proof. The existence of the representation (5) is by Theorem 2.4 equivalent to the $\hat{\tau}$-continuity of the functional $\mathfrak{\Xi}=\left(1, \mathfrak{\Xi}_{1}, \Xi_{2}, \ldots\right)$. The necessity of (6), i.e. 
the proof that the distribution estimate in Theorem 3.7 leads to the estimate (6) for the function $\Xi_{n}$ can be shown in the same way as in [17], Theorem 4.1.

The converse statement follows from Theorem 3.7 because

$$
r\left(x_{i_{1}} \ldots x_{i_{v+1}}\right) \leqq\left|x_{i_{v}}-x_{i_{v+1}}\right| \leqq d\left(x_{i_{1}} \ldots x_{i_{v+1}}\right)
$$

implies

$$
\begin{aligned}
& \sum_{v} \sum_{i_{1}<\ldots<i_{v}}\left(d\left(x_{i_{1}} \ldots x_{i_{v}}\right)^{-k_{v}}+r\left(x_{i_{1}} \ldots x_{i_{v}}\right)^{l_{v}}\right) \\
& \leqq \sum_{\nu=1}^{n} \sum_{v \leqq i<j}\left(\frac{1}{\left|x_{i}-x_{j}\right|^{k_{v+1}}}+\left|x_{i}-x_{j}\right|^{l_{\nu+1}}\right) .
\end{aligned}
$$

This last expression evidently satisfies the condition of Theorem 3.7.

Remarks 1) The estimate (6) says in particular that when any $v$ points $x_{i_{1}} \ldots x_{i_{v}}$ come together, the singularity is controlled by the exponent $k_{v}$ which is independent of the other variables and also of $n$. As remarked earlier, this property seems to be rather closely connected with the existence of a Wilson-Zimmermann expansion, in any case the existence of such an expansion is a sufficient condition.

2) As to the possibility of proving the estimates (6) from the Wightman axioms we note that if all the points of the group $x_{i_{1}} \ldots x_{i_{v}}$ have larger (or smaller) time coordinates than the remaining points one can by permutation bring this group to one end in the argument of the Schwinger function and apply Schwarz inequality to show that the singularity produced by these points is dominated by the $2 v$ point function. This is however only a first step and it is unclear whether the estimates hold in general.

3) In order to connect the representation (6) with a commutative field theory one can pass from the complex measure $d \mu$ to the positive measure $d v=d|\mu|\left(\int d|\mu|\right)^{-1}$ and construct a Hilbert space $L_{2}\left(\mathscr{S}_{R}^{\prime}, d v\right)$ and a cyclic representation $f \rightarrow \phi(\underline{f})$ of $S(\mathscr{S})$ with the cyclic unit vector $\Omega_{0}=1$. The Schwinger functions can then be written as an expectation value

$$
\mathfrak{S}\left(x_{1}, \ldots, x_{n}\right)=\left\langle\Omega_{0}, \phi\left(x_{1}\right) \ldots \phi\left(x_{n}\right) M \Omega_{0}\right\rangle
$$

where $M$ is a metric operator defined as multiplication with the function $f(\omega)=$ $\frac{d \mu_{\omega}}{d v_{\omega}}$. $M$ obviously commutes with all $\phi(x)$, and $\|M\|=|f|=\left(\int d|\mu|\right)$.

4) Nothing has been said about additional conditions which one might like to impose on the measure, e.g. invariance under the Euclidian group. At this place we would only like to make a few comments. Given a measure one can always obtain a rotation invariant one by integrating over the compact rotation group. However, it is clear that if the Schwinger functions shall have a representation by a translation invariant measure they must be bounded as distributions, i.e. the estimate (6) must hold with $l_{v}=0$. This is so because every invariant measure is a linear combination of positive invariant measures, and every strongly positive invariant functional on $S(\mathscr{S})$ satisfies the estimate of Theorem 3.7 with invariant seminorms $p_{v}$ as follows from repeated application of Schwarz inequality. 


\section{References}

1. Borchers, H. J., Yngvason, J.: Commun. math. Phys. 43, 255 (1975)

2. Challifour, J., Slinker, S.: Commun. math. Phys. 43, 41 (1975)

3. Hegerfeldt, G.C.: Extremal Decompositions of Wightman Functions and States on Nuclear *-algebras by Choquet Theory. Commun. math. Phys. 45, 133-135 (1975)

4. Simon, B.: Helv. Phys. Acta 46, 686 (1973)

5. Schlieder, S., Seiler,E.: Commun. math. Phys. 31, 137 (1973)

6. Baumann, K.: Commun. math. Phys. 43, 73 (1975)

7. Collella, P., Lanford, O. E., III: Sample Field Behaviour for the free Markov Field, in: Constructive Quantum Field Theory (ed. G. Velo, A. Wightman). Berlin-Heidelberg-New York: Springer 1973

8. Schaefer,H.H.: Topological Vector Spaces. New York-Heidelberg-Berlin: Springer 1971

9. Wyss, W.: Commun. math. Phys. 27, 223 (1972)

10. Maurin, K.: General Eigenfunction Expansion and Unitary Representation of Topological Groups. Warszawa: Polish Scientific Publishers 1968

11. Gel'fand, I. M., Vilenkin, N. Ya.: Generalized Functions, Vol. 4. New York: Academic Press 1964

12. Boas, R.P.: Bull. Am. Math. Soc. 45, 399 (1939)

13. Lassner, G.: Rep. Math. Phys. 3, 279 (1972)

14. Pietsch, A.: Nuclear Locally Convex Spaces. Berlin-Heidelberg-New York: Springer 1972

15. Yngvason, J.: Commun. math. Phys. 34, 315 (1973)

16. Osterwalder, K., Schrader, R.: Commun. math. Phys. 31, 83 (1973)

17. Osterwalder, K., Schrader, R.: Commun. math. Phys. 42, 281 (1975)

Communicated by K. Hepp

Received October 17, 1975 
